\title{
Regionalentwicklung in Mittel- und Osteuropa im Spannungsfeld von Transformation und Integration am Beispiel Polens
}

\author{
Regional Development in Central and Eastern Europe: \\ Between Transformation and Integration. \\ The Example of Poland
}

\begin{abstract}
Kurzfassung
Die mit dem Zusammenbruch der kommunistischen Systeme in Mittel- und Osteuropa ausgelösten Transformationsprozesse haben in den betroffenen Ländern zum einen auf nationaler Ebene im Hinblick auf das Niveau wirtschaftlicher Aktivitäten und zum anderen auf regionaler Ebene im Hinblick auf die Verteilung weitreichende Effekte nach sich gezogen. Am Beispiel Polens wird anhand verschiedener regionalwirtschaftlicher Theorien aufgezeigt, daß trotz einer positiven nationalen Entwicklung die Disparitäten auch mittelfristig weiter ansteigen werden und erst langfristig eine konvergente Entwicklung wahrscheinlich ist. Auch die sich erst langsam entwickelnde nationale Regionalpolitik sowie die zunehmende Unterstützung durch die EU-Regionalpolitik wirken der divergenten Entwicklung nur wenig entgegen, da die nationale Konvergenz im Vergleich zu den Mitgliedstaaten der EU politische Priorität aufweist.
\end{abstract}

\begin{abstract}
The transformation processes unleashed in Central and Eastern Europe with the collapse of the communist system have had far-reaching effects on the countries concerned: both at national level in respect of the level of economic activity, and at regional level with regard to distribution. Taking Poland as an example, this article draws on a number of theories of regional economy to show how disparities will be heightened over the medium term, and that convergence is only likely in the long term. Neither national regional policy, which is only slowly starting to get off the ground, nor the increasing support provided by EU regional policy is capable of offsetting divergent development, as political priority is given to national convergence vis-àvis EU member states.
\end{abstract}

\section{Einführung}

Die mit dem Zusammenbruch der kommunistischen Systeme in Mittelund Osteuropa ausgelösten Transformationsprozesse haben in den betroffenen Ländern zum einen auf nationaler Ebene im Hinblick auf das Niveau wirtschaftlicher Aktivitäten und zum anderen auf regionaler Ebene im Hinblick auf die Verteilung weitreichende Effekte nach sich gezogen. Die Erfahrungen aus früheren Erweiterungen der EU lassen zudem erwarten, daß die nächsten Erweiterungsrunden in Richtung Osten, die für die erste Dekade des neuen Jahrtausends anstehen, zu weiteren Spannungen führen könnten. Wie sich die beiden Aufgaben der Transformation und der Integration auf die Regionalentwicklung der Kandida- tenländer der EU auswirken, soll im folgenden am Beispiel Polens untersucht werden.

Nach einem kurzen Überblick über den Stand der Transformation auf nationaler Ebene (Kap. 2) werden zunächst die Regionalentwicklung empirisch in ihren Phasen seit dem Umschwung beschrieben, mögliche Theorieansätze zur Erklärung der Entwicklungen aufgezeigt und eine Interpretation der tatsächlichen Entwicklungen geliefert (Kap. 3). Da die Regionalentwicklung nicht nur durch die (privat-)wirtschaftliche Entwicklung, sondern auch durch die Politik bestimmt wird, folgt eine Darstellung der regionalpolitischen Potentiale von der EU- bis zur regionalen Ebene (Kap. 4). Abschließend werden Zukunftsperspektiven der regionalen Entwicklung, insbesondere im
Hinblick auf die Integration der mittelund osteuropäischen Länder (MOEL) in die Europäische Union, skizziert und Empfehlungen für die Regionalpolitik auf den verschiedenen Ebenen abgeleitet (Kap. 5).

\section{Der Entwicklungsstand der MOEL}

Für einen Ländervergleich erscheint das am EU-Durchschnitt der 15 Mitgliedstaaten für das Jahr 1997 standardisierte Bruttoinlandsprodukt je Einwohner (BIP/EW) in Kaufkraftparitäten (KKP) als der geeignetste Indikator, da er die relativen Preise in den jeweiligen Ländern berücksichtigt.' Bei der Betrachtung des BIP/EW in KKP läßt sich feststellen, daß das 
Tabelle 1

Basisdaten der MOEL und der EU15 für das Jahr 1997

\begin{tabular}{|c|c|c|c|c|c|c|c|c|}
\hline & \multirow{2}{*}{$\begin{array}{c}\text { Fläche } \\
1000 \mathrm{~km}^{2}\end{array}$} & \multicolumn{2}{|c|}{ Bevölkerung } & \multicolumn{2}{|c|}{$\mathrm{BIP}(\mathrm{KKP})^{\prime}$} & \multicolumn{2}{|c|}{ Agrarsektor $^{3}$} & \multirow{2}{*}{$\begin{array}{c}\text { Arbeits- } \\
\text { losigkeit }\end{array}$} \\
\hline & & Mio. & $\mathrm{EW} / \mathrm{km}^{2}$ & $\mathrm{ECU} / \mathrm{EW}^{2}$ & $\begin{array}{c}\text { je EW } \\
\text { EU15=100 }\end{array}$ & $\begin{array}{c}\% \text { der ge- } \\
\text { samten BWS }\end{array}$ & $\begin{array}{l}\% \text { der Be- } \\
\text { schäftigten }\end{array}$ & \\
\hline Ungarn & 93 & 10.1 & 109 & 8900 & 47 & 6,7 & 7,9 & 8.1 \\
\hline Polen & 313 & 38.7 & 124 & 7500 & 39 & 5,9 & 20,5 & 11.2 \\
\hline Rumänien & 238 & 22.5 & 94 & 5800 & 31 & 20,1 & 39,0 & 6.0 \\
\hline Slowak. Rep. & 49 & 5.4 & 110 & 8900 & 47 & 6,0 & 8,6 & 11.6 \\
\hline Lettland & 65 & 2.5 & 38 & 5100 & 27 & 7,4 & 18,3 & 14.4 \\
\hline Estland & 45 & 1.5 & 32 & 7000 & 37 & 6,3 & 9,9 & 10.5 \\
\hline Litauen & 65 & 3.7 & 57 & 5800 & 31 & 12,7 & 21,9 & 14.1 \\
\hline Bulgarien & 111 & 8.3 & 75 & 4400 & 23 & 15,4 & 24,4 & 15.0 \\
\hline Tschechien & 79 & 10.3 & 131 & 12000 & 63 & 5,0 & 5,8 & 4.7 \\
\hline Slowenien & 20 & 2.0 & 98 & 13000 & 68 & 4,4 & 10,1 & 7.3 \\
\hline Belgien & 31 & 10.1 & 333 & 21470 & 113 & 1.7 & 2.7 & 9.2 \\
\hline Dänemark & 43 & 5.2 & 122 & 21850 & 115 & 3.7 & 4.4 & 5.5 \\
\hline Deutschland & 357 & 80.6 & 226 & 21090 & 111 & 1.0 & 3.2 & 10.0 \\
\hline Griechenland & 132 & 10.3 & 78 & 12920 & 68 & 14.7 & 20.4 & 9.6 \\
\hline Spanien & 506 & 38.9 & 77 & 14820 & 78 & 3.7 & 9.3 & 20.8 \\
\hline Frankreich & 544 & 56.8 & 104 & 19760 & 104 & 2.5 & 4.9 & 12.4 \\
\hline Irland & 70 & 3.6 & 51 & 18620 & 98 & 7.5 & 12.0 & 10.1 \\
\hline Italien & 301 & 56.6 & 188 & 18810 & 99 & 2.9 & 7.5 & 12.1 \\
\hline Luxemburg & 3 & 0.4 & 146 & 30780 & 162 & 1.5 & 3.9 & 2.6 \\
\hline Niederlande & 42 & 15.3 & 368 & 20140 & 106 & 3.6 & 3.8 & 5.2 \\
\hline Österreich & 84 & 7.9 & 94 & 21280 & 112 & 2.4 & 7.3 & 4.4 \\
\hline Portugal & 92 & 9.8 & 107 & 13300 & 70 & 5.1 & 11.5 & 6.8 \\
\hline Finnland & 338 & 5.1 & 15 & 18620 & 98 & 5.2 & 7.8 & 13.1 \\
\hline Schweden & 450 & 8.8 & 20 & 19000 & 100 & 2.1 & 3.3 & 9.9 \\
\hline Ver. Königreich & 244 & 57.8 & 239 & 18810 & 99 & 1.6 & 2.1 & 7.0 \\
\hline
\end{tabular}

1 Kaufkraftparitäten (KKP) werden üblicherweise anstelle der jeweiligen amtlichen Wechselkurse benutzt, um den relativen Lebensstandard zu schätzen. Sie berücksichtigen daher Kostenunterschiede zwischen den Ländern.

2 BIP/EW in KKP für die MOEL: Überarbeitete Daten, für welche die neuen KKP-Ergebnisse des Internationalen Vergleichsprojektes 1996 verwendet werden (OECD, Eurostat, nationale Statistische Ämter).

3 Daten für den Agrarsektor: EU15 für 1995

Quelle: eigene Zusammenstellung in freundlicher Zusammenarbeit mit Friedrich Schindegger, ÖIR, Wien, aus Europäische Kommission, 1998: Regelmäßige Berichte der Kommission ïber die Fortschritte auf dem Weg zum Beitritt. Brüssel (einzelne Länderberichte, Internetversionen); European Commission, 1997: Agenda 2000, S. 91; EUROSTAT, 1999: Eurostatistik, Daten zur Konjunkturanalyse - Themenkreis 1, Reihe B, Brüssel; EUROSTAT, 1999: Statistisches Jahrbuch für die MOEL, Brüssel

Land mit dem relativ höchsten Wohlstandsniveau der MOEL (Slowenien) mit $68 \%$ des EU-Durchschnitts an das Land mit dem niedrigsten BIP/EW innerhalb der EU (Griechenland) mit $69 \%$ heranreicht (Tab. 1).

Innerhalb der MOEL erreicht nur Tschechien das $60 \%$-Niveau. Diese
Gruppe wird gefolgt von der Slowakei, Polen und Ungarn im Bereich von 40 bis $47 \%$ des EU-Durchschnitts. Die baltischen Staaten sowie Rumänien und Bulgarien folgen mit 23-37\% des EU-Durchschnitts. Damit wird deutlich, daß auch innerhalb der MOEL ein hohes Gefälle besteht. Die hohen An- teile des Agrarsektors in den MOEL deuten zudem auf den großen Nachholbedarf im Bereich des wirtschaftlichen Strukturwandels hin. ${ }^{2}$

In einigen MOEL bestehen noch Schwierigkeiten, wichtige makroökonomische Größen wie die Inflationsrate oder das Staatsdefizit zu stabili- 
Abbildung 1

BIP-Wachstum in den MOEL 1989-1998 (reales Wachstum in \%)

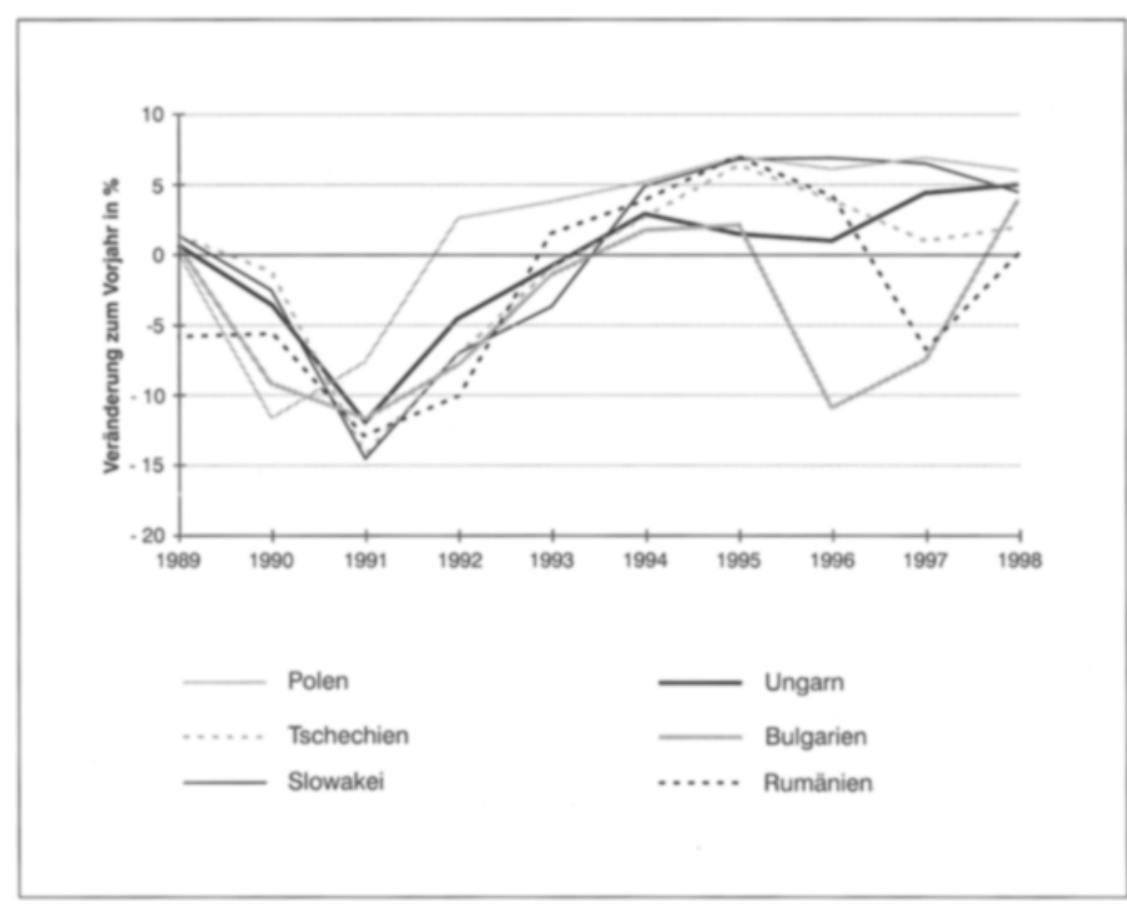

Quelle: European Bank for Reconstruction and Development (EBRD) 1995: Transition Report; Bundesministerium für Wirtschaft 1995, 1998: Wirtschaftslage und Reformprozesse in Mittel- und Osteuropa. Sammelband

sieren, allerdings pendeln sich die Wachstumsraten nach einem starken Rückgang auf einem Niveau von 2-7\% ein, wobei Bulgarien und $\mathrm{Ru}-$ mänien 1996 bzw. 1997 insofern Ausnahmen bildeten, als in beiden Ländern das BIP gegenüber dem Vorjahr schrumpfte (Abb. 1). Dies ist zum einen auf den grundlegenden wirtschaftlichen Strukturwandel, der in den einzelnen Staaten in unterschiedlicher Weise notwendig ist, und zum anderen auf ein unterschiedliches Tempo bei den institutionellen Reformen $\mathrm{zu}$ marktwirtschaftlichen Institutionen zurückzuführen.

Ein Überblick der EBRD (1997) zeigt deutlich, daß die Transformation in bezug auf die Privatisierung bei Kleinunternehmen sowie die Liberalisierung im Handel und auf den Devisenmärkten am weitesten fortgeschritten ist und nahezu als abgeschlossen bezeichnet werden kann (Tab. 2). Gerade in den Visegradstaaten und in Slowenien ist ein hoher Stand erreicht; auch die baltischen Staaten haben hier sehr groBe Fortschritte erzielt. Im Vergleich dazu bestehen jedoch in fast allen Ländern noch Defizite in der Privatisierung großer Unternehmen. Hier wirkt die sozialistische Strategie der Förderung von Schwerindustrie in großen Unternehmen nach. Erschwerend kommt bei der Privatisierung der Großunternehmen hinzu, daß die Verfahren oft wenig transparent ausgestaltet sind und somit für die Bevölkerung wenig nachvollziehbar bleiben. Im Hinblick auf die Liberalisierung der Preise haben die meisten Staaten die Kontrolle vollständig aufgehoben. Allerdings wurden gerade von weniger weit fortgeschrittenen Staaten bei besonderen Problemlagen (z. B. Bulgarien) selektive Preiskontrollen wieder eingeführt.

Insbesondere besteht noch Reformbedarf im Finanzsektor als grundlegendem Element der Marktsteuerung. Nach dem Aufbau eines zweistufigen Bankensystems wurden im Bereich der Geschäftsbanken bisher nicht sehr viele Privatisierungen vorgenommen. Makroökonomische Schocks und wirtschaftliche Unsicherheiten haben Ban- ken immer wieder dazu veranlaßt, langfristige Kredite zu verweigern. Zur Ermöglichung von Investitionen auf der einen Seite müssen auf der anderen Seite Sparguthaben aufgebaut werden. Allerdings sind Sparformen wie Lebensversicherungen, Pensionsfonds und ähnliches in den MOEL noch wenig entwickelt, weshalb der Kreislauf von Sparen und Investieren schwer in Gang kommt. ${ }^{3}$ Die Regierung Polens hat z. B. im Sommer 1998 die mittelfristige Strategie der Öffentlichen Finanzen vor dem polnischen Parlament präsentiert. Diese Strategie des „nachhaltigen Wachstums“ sieht vor, den BIP-Anteil der Investitionen zu steigern, da mittlerweile das Anlagekapital aus der sozialistischen Zeit weitestgehend verbraucht ist. Eine derartige Investitionssteigerung kann nach Ansicht der Regierung nur durch sinkende Steuerbelastungen und verringerte öffentliche Ausgaben erzielt werden. Andererseits wird empfohlen, die Liberalisierung des Kapitalsektors weiter voranzutreiben, so daß zukünftig verstärkt internationales Investitionskapital (hot money) nach Polen fließen kann. ${ }^{4}$

\section{Regionale Disparitäten}

\subsection{Entwicklung der regionalen Disparitäten}

Nun soll der Blick auf die Regionen ausgewählter MOEL konzentriert werden. Hinsichtlich des hier verwendeten Indikators BIP/EW in KKP ist darauf hinzuweisen, daß für die betrachteten MOEL nur für ein Jahr (1993) Daten vergleichbar mit der NUTS I-Ebene verfügbar sind (Abb. 2). ${ }^{6}$

Werden die regionalen Disparitäten in den MOEL im Zusammenhang mit der EU und den dort vorliegenden Disparitäten gesehen, so ergibt sich ein relativierendes Bild in folgender Weise. ${ }^{7}$ Die besser entwickelten Regionen in den MOEL überschreiten durchaus die Landesdurchschnitte in den ärmeren Mitgliedstaaten. Insofern kann keineswegs davon gesprochen werden, daB auf der regionalen Ebene der betroffenen Staaten kein Anschluß an das EU- 
Tabelle 2

Transformationsstand in ausgewählten MOEL (1997)

\begin{tabular}{|l|c|c|c|c|c|c|c|c|c|}
\hline \multirow{2}{*}{} & \multicolumn{3}{|c|}{} & \multicolumn{3}{c|}{ Märkte, Handel } & \multicolumn{2}{c|}{ Finanzinstitutionen } & $\begin{array}{c}\text { Rechts- } \\
\text { reformen* }\end{array}$ \\
\cline { 2 - 9 } Land & $\begin{array}{c}\text { Privatisie- } \\
\text { rung von } \\
\text { Großunter- } \\
\text { nehmen }\end{array}$ & $\begin{array}{c}\text { Privatisie- } \\
\text { rung von } \\
\text { Kleinunter- } \\
\text { nehmen }\end{array}$ & $\begin{array}{c}\text { Leitung } \\
\text { und Re- } \\
\text { struktu- } \\
\text { rierung }\end{array}$ & $\begin{array}{c}\text { Liberalisie- } \\
\text { rung der } \\
\text { Preise }\end{array}$ & $\begin{array}{c}\text { Außenhan- } \\
\text { del und } \\
\text { Devisen- } \\
\text { märkte }\end{array}$ & $\begin{array}{c}\text { Wett- } \\
\text { bewerbs- } \\
\text { politik }\end{array}$ & $\begin{array}{c}\text { Bankreform, } \\
\text { Zinslibera- } \\
\text { lisierung }\end{array}$ & $\begin{array}{c}\text { Sicherheit, } \\
\text { Finanz- } \\
\text { bereich der } \\
\text { Nichtbanken }\end{array}$ & $\begin{array}{c}\text { Deregulie- } \\
\text { rung, Effi- } \\
\text { zienzstei- } \\
\text { gerung bei } \\
\text { Investitionen }\end{array}$ \\
\hline Tschechien & 4 & $4+$ & 3 & 3 & $4+$ & 3 & 3 & 3 & 4 \\
Ungarn & 4 & $4+$ & $3+$ & $3+$ & $4+$ & 3 & 4 & $3+$ & 4 \\
Polen & $3+$ & $4+$ & 3 & $3+$ & $4+$ & 3 & $3+$ & $3+$ & 4 \\
Slowakei & 4 & $4+$ & $3-$ & 3 & $4+$ & 3 & $3-$ & $2+$ & 2 \\
Slowenien & $3+$ & $4+$ & $3-$ & 3 & $4+$ & 2 & 3 & 3 & 3 \\
Bulgarien & 3 & 3 & $2+$ & 3 & 4 & 2 & $3-$ & 2 & 4 \\
Rumänien & $3-$ & $3+$ & 2 & 3 & 4 & 2 & $2+$ & 2 & 4 \\
Estland & 4 & $4+$ & 3 & 3 & 4 & $3-$ & $3+$ & 3 & 3 \\
Litauen & 3 & 4 & $3-$ & 3 & 4 & $2+$ & 3 & $2+$ & 3 \\
Lettland & 3 & 4 & $3-$ & 3 & 4 & $3-$ & $3-$ & $2+$ & 3 \\
\hline
\end{tabular}

* Durchschnittswert

Quelle: Eigene Zusammenstellung aus European Bank for Reconstruction and Development (EBRD) Transition Report 1998, S. 26, 42

Niveau besteht; dies betrifft insbesondere die Hauptstadtregionen dieser Länder. Umgekehrt zeigt sich jedoch auch, daß - trotz relativ geringer regionaler Extremwerte nach unten - bestimmte Regionen in diesen Ländern ein äußerst niedriges Wohlstandsniveau im Vergleich zur EU aufweisen.

Dieses im internationalen Zusammenhang relativierte Bild kann nicht darüber hinwegtäuschen, daß innerhalb der Länder teilweise große Disparitäten vorliegen. ${ }^{8}$ Auffällig ist zunächst einmal, daß die Hauptstadtregionen in der Regel den nationalen Durchschnitt des BIP pro Einwohner in Kaufkraftparitäten weit übertreffen, wie dies beispielsweise bei Bukarest (um $25 \%$ ), Prag (um 50\%), Warszawa (um $70 \%$ ), Budapest (um $80 \%$ ) und Sofia (sogar um $150 \%$ ) der Fall ist. Umgekehrt ist in den meisten Ländern die Abweichung vom nationalen Durchschnitt nach unten relativ gering. So weicht die ärmste Region Slowakiens (Zentralslowakien) um $11 \%$, Tschechiens (Nordböhmen) um $13 \%$ und Rumäniens (Nordmoldau) ebenfalls um $13 \%$ nach unten ab. Größere
Disparitäten bestehen allerdings in Ungarn (Nordostregion) mit $32 \%$ und Bulgarien (Montana) mit $50 \%$ negativer Abweichung. Nur in Bulgarien überschreiten über die Hälfte der Regionen eine negative Abweichung nach unten von über $35 \%$. Allerdings muß hier berücksichtigt werden, daß die Landeshauptstadt mit einer Überschreitung des Durchschnitts um $150 \%$ diesen stark nach oben zieht.

Ein genaueres Bild der Entwicklung der regionalen Disparitäten hinsichtlich des BIP/EW soll am Beispiel Polens gezeichnet werden, wobei zwei Transformationsphasen, der Zusammenbruch (1986-1992) und der Aufschwung des planwirtschaftlichen Systems (1992-1995), unterschieden werden. Da die Werte in den polnischen Regionen nur in Relation zum jeweiligen nationalen Durchschnitt $(=100)$ berechnet wurden, ist ein Vergleich mit anderen Regionen der MOEL nicht möglich.

Die regionalen BIP/EW-Werte des Zeitraums von 1986-1992 deuten darauf hin, daß die regionalen Disparitäten in Polen in der ersten Phase der
Transformation, die sich durch einen insgesamt starken Produktionsrückgang auszeichnet, leicht abgenommen haben. ${ }^{9}$ Dies kann im wesentlichen auf die Einbußen im BIP/EW der Wojewodschaft Warszawa zurückgeführt werden. Liegt das BIP/EW 1986 dort noch $80 \%$ über dem polnischen Durchschnitt, so ist dieses Plus bis 1992 auf $58 \%$ abgesunken. Obwohl neben den Verlusten von Warszawa vier weitere Agglomerationen (Katowice, Lódz, Wróclaw, Gdansk) von 1986-1992 relative Verluste verzeichnen (Tab. 3), gehören sowohl 1986 als auch 1992 die polnischen Agglomerationen zu den reichsten Regionen des Landes.

Im Unterschied zur dynamischen Entwicklung in der Gruppe der reichsten Regionen haben sich die ärmsten Regionen von 1986-1992 kaum dem polnischen Durchschnitt angenähert. Die agrarisch dominierten Wojewodschaften des Ostens zählen zu den ärmsten Regionen in Polen. Im westlichen Landesteil zeigt sich in der ersten Transformationsphase eine stärkere Entwicklungsdynamik als im polnischen Osten. Hier liegen stark wachsende 
Abbildung 2

Spannweite der regionalen Daten (NUTS I) zum Bruttoinlandsprodukt je Einwohner in Kaufkraftparitäten für die EU-Staaten, Visegrad sowie Rumänien und Bulgarien für das Jahr 1993 (EU15 = 100)

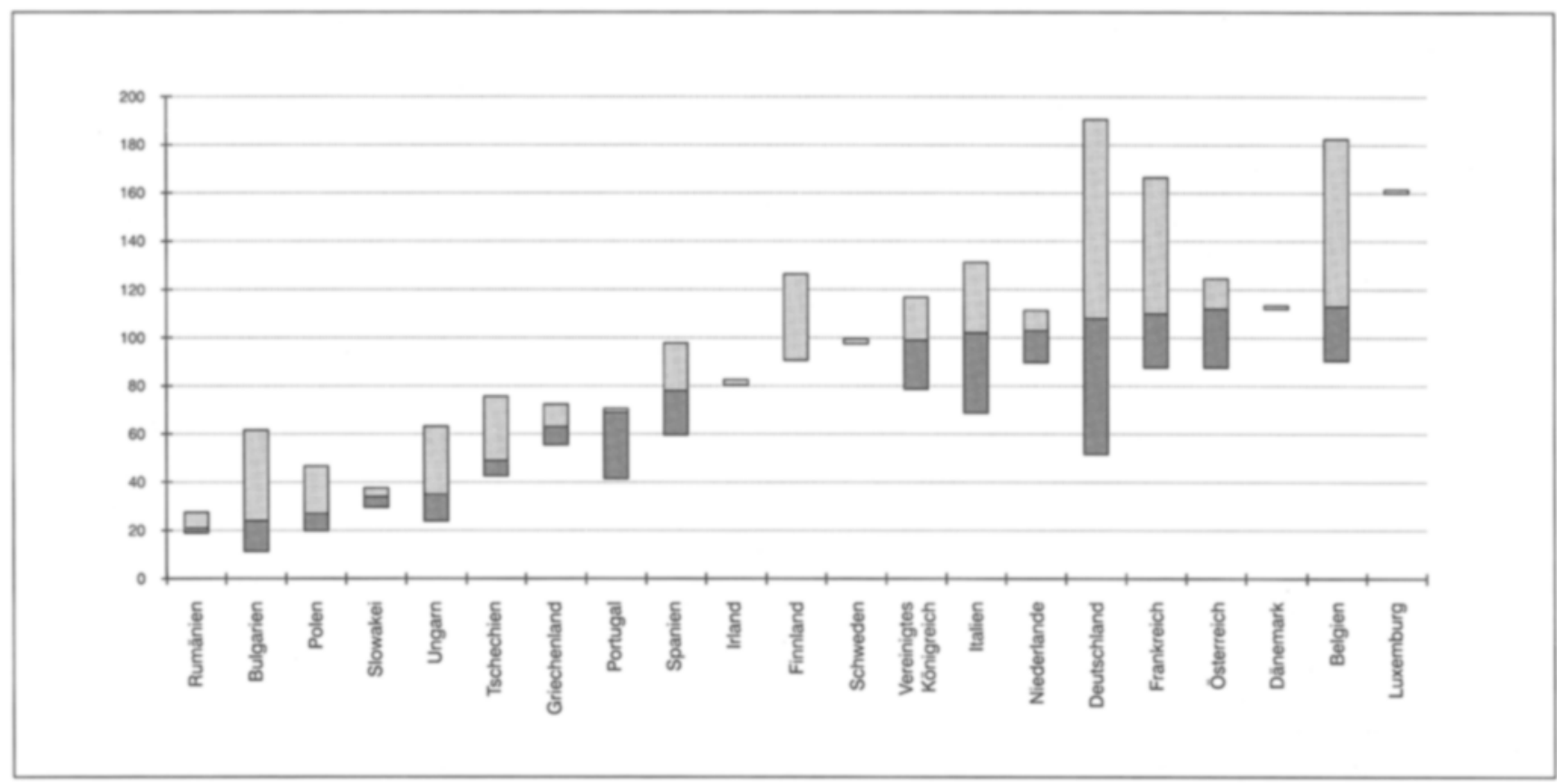

Quelle: Eser 1998, EPRC/RWI 1996

Abbildung 3

Polnische Regionen (Wojewodschaften)

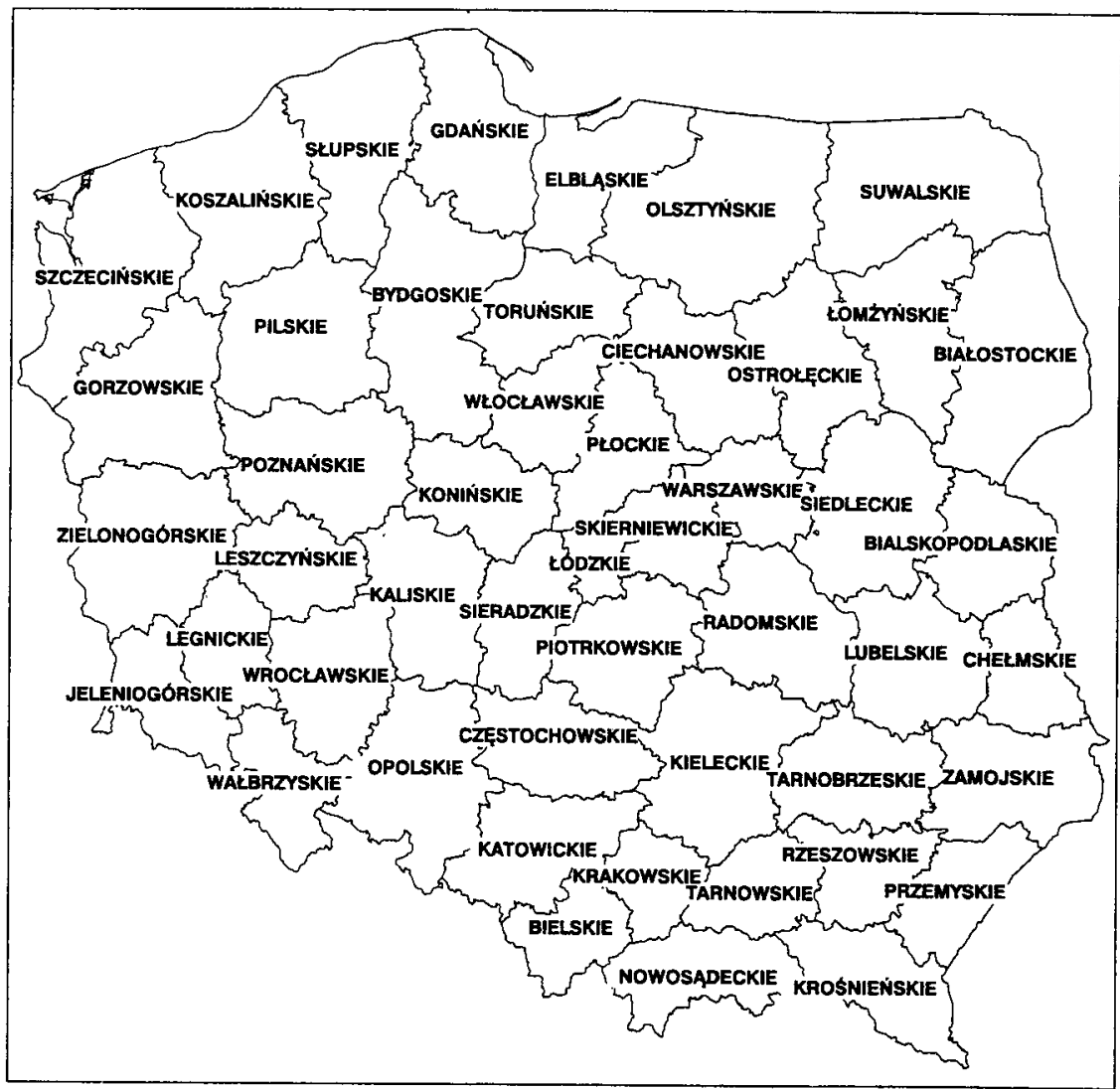

Quelle: Gorzelak 1998, S. 6
Regionen in unmittelbarer räumlicher Nähe zu stagnierenden Wojewodschaften (z. B. Bydgoszcz und Gdansk). Die größere Dynamik des Westens zeigt sich auch darin, daß drei der vier Regionen, die 1986 auf einem unterdurchschnittlichen Entwicklungsniveau liegen und bis 1992 auf ein überdurchschnittliches Niveau gestiegen sind, im westlichen Polen liegen.

1992 kehrt sich der negative Entwicklungsproze $\beta$ um, und die polnische Wirtschaft wächst erstmals wieder seit dem Beginn der Transformation $(+2,6 \%)$. Seit 1995 betragen die jährlichen Wachstumsraten 6-7 \%. In dieser zweiten Transformationsphase sind die regionalen Disparitäten (BIP/EW) von 2,5:1 (1992) auf 3,5:1 (1995) angestiegen. Die relativen Veränderungen der regionalen BIP/EW-Daten deuten jedoch auf kein eindeutiges räumliches Muster hin (Tab. 3). Einerseits verzeichnen einige Agglomerationen (Warszawa, Wróclaw) ein hohes relatives Wachstum, andererseits haben sich die Agglomerationen Kraków, Poznan und Szczecin auf- 
grund ihrer relativen Verluste an den polnischen Durchschnitt angenähert. Während einige Industrieregionen, bei denen man aufgrund der sinkenden Nachfrage nach Rohstoffen starke Verluste befürchten konnte, gewachsen sind (Katowice, Konin), ist das $\mathrm{BIP} / \mathrm{EW}$ in Relation zum nationalen Durchschnitt in anderen industriell geprägten Wojewodschaften (z. B. Legnica) gesunken. Ein ebenso uneinheitliches Bild zeigt sich in den östlichen Regionen Polens. Hier entwickelt sich z. B. Ostroleka positiv, während andere Wojewodschaften (Chelm, Biala Podlaska, Zamosc) starke Einbußen verzeichnen mußten. ${ }^{10}$
Die polnische Regionalentwicklung deutet darauf hin, daß die regionalen Disparitäten in der Transformationsphase angestiegen sind. Dabei bildeten sich einerseits ein Ost-West-Gefälle und andererseits ein Stätt-Land-Gefälle deutlich heraus. Allerdings haben sich weder die Agglomerationen noch die agrarisch geprägten östlichen Wojewodschaften in Polen in diesem Prozeß einheitlich entwickelt. Während Warszawa und Katowice in der ersten Phase eine negative Entwicklung des $\mathrm{BIP} / \mathrm{EW}$ verzeichneten, kehrte sich dieser Prozeß nach 1992 in eine positive Richtung um. Die schwerindustriell dominierte und unter erheblichen Um- weltproblemen leidende Wojewodschaft Katowice überrascht in zweifacher Hinsicht: zum einen durch die positive Entwicklung des BIP/EW nach 1992 und zum anderen durch die geringe Arbeitslosenrate (1996: $8,4 \%$ ). Ebenso überraschend kehrte sich die BIP/EW-Entwicklung in den Agglomerationen Poznan, Szczecin, Kraków und Bydgoszcz nach einer ersten positiven Entwicklungsphase nach 1992 in eine negative Richtung um. Dabei darf aber nicht vergessen werden, daß diese Regionen auch 1995 noch wesentlich über dem polnischen Durchschnitt lagen. Zwar hat sich im Zeitraum von 1986 bis 1995 der polni-

Tabelle 3

Relative Wachstumsraten des BIP/EW in den polnischen Wojewodschaften ${ }^{11}$

\begin{tabular}{|l|c|c|c|}
\hline Wojewodschaft & $\begin{array}{c}\text { BIP/ } \\
\text { EW 1992 }\end{array}$ & $\begin{array}{c}\text { Relatives } \\
\text { Wachstum } \\
1986-1992\end{array}$ & $\begin{array}{c}\text { Relatives } \\
\text { Wachstum } \\
1992-1995\end{array}$ \\
\hline Warszawa (A) & 158,1 & $-22,0$ & 9,5 \\
Plock & 149,3 & 46,0 & $-7,6$ \\
Poznan (A) & 132,0 & 30,3 & $-19,2$ \\
Szczecin (A) & 126,7 & 21,4 & $-8,1$ \\
Legnica & 126,4 & 4,8 & $-12,8$ \\
Piotrkow & 120,0 & 29,9 & $-19,9$ \\
Bydgoszcz (A) & 117,2 & 20,5 & $-4,1$ \\
Kraków (A) & 117,2 & 14,7 & $-12,4$ \\
Katowice (A) & 116,1 & $-23,5$ & 15,0 \\
Gdansk (A) & 105,4 & $-2,3$ & 3,0 \\
Lódz (A) & 105,3 & $-4,1$ & 2,2 \\
Zielona Gora & 105,1 & 14,9 & $-11,9$ \\
Wróclaw (A) & 104,9 & $-4,8$ & 14,5 \\
Opole & 100,1 & 2,1 & $-4,3$ \\
Jelenia Gora & 99,2 & 9,9 & $-9,5$ \\
Olsztyn & 91,7 & 6,4 & $-8,5$ \\
Elblag & 88,8 & 3,0 & 4,6 \\
Lublin & 88,6 & $-4,9$ & 3,6 \\
Bielsko Biala & 88,2 & $-3,4$ & 2,5 \\
Leszno & 88,1 & 14,0 & 8,4 \\
Chelm & 87,0 & 16,5 & $-20,3$ \\
Czestochowa & 87,0 & 7,0 & 0,1 \\
Koszalin & 86,1 & $-1,8$ & $-3,4$ \\
Torun & 86,1 & $-5,5$ & $-1,7$ \\
Gorzów & 85,8 & $-1,4$ & 4,5 \\
\hline
\end{tabular}

\begin{tabular}{|l|c|c|c|}
\hline Wojewodschaft & $\begin{array}{c}\text { BIP/ } \\
\text { EW 1992 }\end{array}$ & $\begin{array}{r}\text { Relatives } \\
\text { Wachstum } \\
1986-1992\end{array}$ & $\begin{array}{c}\text { Relatives } \\
\text { Wachstum } \\
1992-1995\end{array}$ \\
\hline Tarnow & 85,4 & 12,6 & $-12,1$ \\
Bialystok & 84,1 & $-1,9$ & $-4,4$ \\
Rzeszow & 82,2 & $-3,6$ & $-2,8$ \\
Tarnobrzeg & 81,2 & $-11,0$ & 7,1 \\
Konin & 80,7 & 1,0 & 13,2 \\
Pila & 80,7 & 4,1 & 11,3 \\
Kalisz & 80,6 & 2,1 & 5,4 \\
Walbrzych & 79,9 & $-13,5$ & $-0,8$ \\
Biala Podlaska & 79,1 & 10,8 & $-7,0$ \\
Ciechanów & 77,7 & 6,6 & $-4,6$ \\
Kielce & 77,5 & $-5,1$ & 3,2 \\
Radom & 77,5 & $-2,9$ & 6,4 \\
Slupsk & 77,4 & $-3,6$ & $-4,9$ \\
Wloclawek & 76,2 & $-3,9$ & $-2,0$ \\
Skierniewice & 73,0 & $-1,8$ & $-0,1$ \\
Zamosc & 72,6 & 4,3 & $-5,6$ \\
Krosno & 72,3 & $-2,3$ & $-3,2$ \\
Siedlce & 71,8 & 4,0 & $-3,7$ \\
Sieradz & 71,7 & 2,5 & 1,3 \\
Lómza & 70,4 & 2,7 & $-3,6$ \\
Ostroleka & 69,9 & 5,6 & 5,6 \\
Przernysl & 68,6 & 1,4 & 1,1 \\
Nowy Sacz & 68,2 & 6,6 & $-4,9$ \\
Suwalki & 64,5 & $-7,8$ & 1,7 \\
& & & \\
\hline
\end{tabular}

(A) = Agglomeration

Quellen: eigene Zusammenstellung nach CUP (1996), Tabelle 18c und Zienkowski (1994), ZBSE (1997), zitiert in Gorzelak (1998), S. 67 
sche Osten tendenziell negativ entwikkelt, jedoch ist seit 1992 ein Differenzierungsproze $\beta$ in dieser Gruppe zu erkennen (z. B. die positive Entwicklung von Ostroleka).

\subsection{Erklärungsansätze \\ zur Entwicklung der regionalen Disparitäten}

Drei Erklärungsansätze für die aufgezeigte Regionalentwicklung sollen hier Berücksichtigung finden, die zum einen die Effekte der Transformation und zum anderen die Integrationseffekte in die westlichen Märkte und in die EU abbilden: erstens die Ausstattung einer Region mit Potentialfaktoren, zweitens der Zusammenhang von Entwicklungsniveau und regionalen Disparitäten und drittens der Zusammenhang zwischen wirtschaftlichem Integrationsgrad einer peripheren Region und ihrem Entwicklungsniveau im Verhältnis zu einer zentralen Region des Integrationsraumes.

Nach dem Potentialfaktorenansatz von Biehl lassen sich die Ressourcen einer Region in Potential- und Produktionsfaktoren einteilen. Für die Entstehung von regionalen Disparitäten sind zwei Aspekte tragend: zum einen die unterschiedliche Ausstattung einer Region mit Potentialfaktoren, zum anderen der Auslastungsgrad des regionalen Potentials. Die „Potentialfaktoren (...) bestimmen mittelfristig das regionale Entwicklungspotential, da sie zu Engpaßfaktoren der Regionalentwicklung werden können“ (und) ,weisen in hohem Maße Eigenschaften öffentlicher Güter (Immobilität, Unteilbarkeit, Polyvalenz, Limitationalität) auf." 12 Der Auslastungsgrad des regionalen Potentials bestimmt sich durch das Maß, in dem die privaten Produktionsfaktoren das Potential nutzen.

$\mathrm{Zu}$ den Potentialfaktoren zählen die wirtschaftsgeographische Lage, der Agglomerationsgrad sowie die Sektor- und Infrastruktur. So bedeutet eine zentrale wirtschaftsgeographische Lage, d. h. die räumliche Nähe zu den
Märkten, niedrige interregionale Kosten für Transporte und Kommunikation für die Unternehmen und Haushalte einer Region. Diese Kosten sind in einer peripheren Lage entsprechend höher. Weiterhin bedingen Siedlungsstruktur und Agglomerationsgrad die Nutzung von Größenvorteilen (economies of scale). $\mathrm{Ab}$ einer gewissen Agglomerationsgröße jedoch schlagen die Vorteile in negative Effekte um. Der gesamtwirtschaftliche Ertrag einer gegebenen Menge und Qualität von Produktionsfaktoren ist dann am höchsten, wenn diese sektoral und branchenmäßig optimal eingesetzt sind. Auch bestimmt die infrastrukturelle Ausstattung einer Region ebenso wie die übrigen genannten Determinanten die Produktivität einer Region. So können durch die Verbessrung der Verkehrsinfrastruktur in eine Region die Transportkosten verringert werden. Neben der Verkehrsinfrastruktur können als weitere infrastrukturelle Bereiche Telekommunikation, Umweltschutz und Bildung genannt werden. ${ }^{13}$

Die Transformationsphase einer Volkswirtschaft kann als eine Periode verstanden werden, in der das regionale BIP sowohl wachsen als auch schrumpfen kann (im Sinne des zweiten hier vorzustellenden Theorieansatzes der Williamson-Hypothese) und das Ausmaß der regionalen Disparitäten einer Volkswirtschaft auf ihren wirtschaftlichen Entwicklungsstand zurückgeführt wird, wobei ein tradeoff zwischen der nationalen Entwicklung und dem regionalen Ausgleich beobachtet wird. Williamson nimmt an, daß die regionalen Disparitäten in einer ersten Phase, die durch ein relativ geringes, aber steigendes BIP gekennzeichnet ist, zunehmen, es aber bei einem ausreichend hohen Wohlfahrtsniveau zur Umkehrung dieses Prozesses kommt und die regionalen BIP konvergieren. Determinanten der regionalen Disparitäten sind die interregionalen Faktorwanderungen von Arbeit und Kapital, die infrastrukturellen Verbindungen zwischen den Regionen sowie die Regionalpolitik.

In einer ersten Phase des volkswirtschaftlichen Wachstums - ausgehend von einem relativ geringen Wohlstandsniveau - kommt es zu den von Myrdal und Hirschmann beschriebenen Entzugs- bzw. Polarisationseffekten durch selektive Migration von Arbeitskräften sowie Kapitalwanderungen in Richtung der reicheren Regionen bzw. nationalen Zentren. Motivationen hierfür sind einerseits höhere Löhne und andererseits eine höhere Kapitalrendite in den Zentren. Der daraus folgende Anstieg der regionalen Disparitäten kann aufgrund des mit einem niedrigen nationalen Wohlstandsniveau korrespondierenden Finanzmangels nicht durch eine regional orientierte Politik abgemildert werden.

Im weiteren folgt die Argumentation von Williamson der neoklassisch orientierten Faktormobilitätstheorie. Die Wanderungen des Faktors Arbeit werden aufgrund des steigenden nationalen Wohlstands weniger selektiv, und die Migration in Richtung der reicheren Regionen wächst an. Auch verbessern sich die institutionellen Bedingungen und die Kapitalrendite in den ärmeren Regionen, so daß auch der Faktor Kapital aus den wohlhabenderen Regionen, unterstützt durch dort steigende Agglomerationsnachteile, abwandert. Die Folge dieser Faktorwanderungen sind Ausgleichsprozesse hinsichtlich der regionalen Einkommen.

Auf einem niedrigen Entwicklungsniveau, d. h. in der ersten Phase, strebt eine zentrale Regierung danach, die nationale Entwicklung zu fördern und unterstïtzt v.a. die Regionen, die als Wachstumspole mit der größten Profitrate angesehen werden. In diese Räume werden die knappen finanziellen Mittel gelenkt. Mit wachsendem nationalen Wohlstand stehen der Regierung zunehmend Mittel zur Beeinflussung der geographischen Verteilung des Einkommens in Form einer aktiven Transferpolitik zugunsten der armen Regionen zur Verfügung. ${ }^{14}$

Die schwachen interregionalen Verbindungen (v. a. die Verkehrs- und Telekommunikationsinfrastruktur) in der ersten Entwicklungsphase verhindern die rasche und umfassende Ausbrei- 
tung von technologischem Fortschritt, sozialem Wandel und Einkommensmultiplikatoren im Raum. Diese Verbindungen werden im wirtschaftlichen Entwicklungsproze $B$ ausgebaut und verstärken in der Folge die Ausbreitungseffekte. ${ }^{15}$

Nach dem letzten hier vorzustellenden Theorieansatz der U-These ${ }^{16}$ bedeutet der Beitritt zu einem gemeinsamen Wirtschaftsraum für die beitretenden Länder die wirtschaftliche Integration als Teilregion in einen Gesamtraum. Aufgrund der geographischen Lage, des Entwicklungsniveaus und der Infrastrukturausstattung im Vergleich zu den EU-Ländern kann davon ausgegangen werden, $\mathrm{da} \beta$ es sich bei den Beitrittsländern um peripher gelegene Regionen handelt. In der wirtschaftswissenschaftlichen Literatur zur Entwicklung von Regionen werden je nach zugrundegelegten Annahmen verschiedene Thesen zur Entwicklung peripherer Regionen bei einer Integration diskutiert ${ }^{17}$ : Grundsätzlich stehen sich die Konvergenzthese, die eine konvergente regionale Entwicklung aufgrund sich annähernder Faktorpreise hervorhebt, und die Divergenzthese, bei der es aufgrund ständiger Entzugseffekte zu einer langfristig divergierenden regionalen Entwicklung kommt, gegenüber.

Die U-These hingegen stellt in gewisser Weise eine Synthese der beiden Ansätze dar, was vor dem Hintergrund der unterschiedlichen Annahmen der Konvergenz- und Divergenzthese nicht zu einem Widerspruch führt. Die U-These basiert auf der Vorstellung, daß die Integration zu einer Reduktion von Tauschkosten führt, wobei Tauschkosten in diesem Sinne jedes Hindernis bei der Durchführung von Tauschvorgängen (wie Zölle, sich ändernde Wechselkurse, Transportkosten oder Kommunikationskosten) umfassen. ${ }^{18}$ Hohe Tauschkosten in einem nicht integrierten Raum behindern den Handel und noch mehr die Mobilität der Produktionsfaktoren zwischen den Teilräumen. Der periphere Markt ist geschützt, wie dies in der Autarkiesituation der Fall ist. Die zunehmende Integration führt zu- nächst zu einer Divergenz des Verhältnisses des Einkommens zwischen Peripherie und Zentrum, da das Zentrum Größenvorteile ausschöpfen kann. Bei weiter abnehmendem Tauschkostenniveau steigt die Attraktivitât der Peripherie im Zeitablauf nicht nur als Absatz-, sondern auch als Produktionsort. Insofern können dann auch in der Peripherie Größenvorteile genutzt werden, indem dort die Produktion für die Märkte im Zentrum stattfindet, womit das Faktorpreisausgleichstheorem der traditionellen Außenhandelstheorie wirksam wird.

\subsection{Interpretation der Regionalentwicklung}

Mittels der aus den drei Erklärungsansätzen resultierenden Determinanten Potentialfaktoren, Faktorwanderungen, Regionalpolitik und Tauschkosten lassen sich nun am Beispiel Polens der Zusammenhang zwischen den Determinanten und der Regionalentwicklung seit dem Beginn der Transformationsphase untersuchen. ${ }^{19}$ Das geringe (seit 1992 kontinuierlich ansteigende) polnische Wohlfahrtsniveau hat zu einer räumlichen Effizienzorientierung geführt, bei der erstens die Agglomerationen, z. B. Warszawa und Poznan, profitierten und zweitens sich besonders die westlichen Regionen positiv entwickelt haben.

Dies ist einerseits auf das positive ,sozialistische Erbe“ dieser Regionen zurückzuführen, d. h. eine günstige Ausstattung mit materieller Infrastruktur (insbesondereVerkehrsinfrastruktur) und eine relativ ausgeglichene Sektorstruktur mit einem Schwerpunkt im Dienstleistungsbereich in den Agglomerationen. Andererseits verfügen diese Regionen über eine günstige wirtschaftsgeographische Lage und eine ausreichend dichte Besiedlung. Ein weiterer Faktor, der v. a. die Entwicklung der Agglomerationen günstig beeinflußt hat, ist ihre relativ gute Ausstattung an Humankapital sowohl bezüglich der Altersstruktur als auch hinsichtlich des Ausbildungsniveaus.
Diejenigen Regionen, die sich im Verlauf des Transformationsprozesses weniger günstig oder sogar negativ entwickelten, sind zum einen durch Altindustrialisierung (Katowice und Walbrzych) und zum anderen durch ihre östliche Lage am sog. eastern wall gekennzeichnet. Bei den altindustrialisierten Regionen sind zwar teilweise die Ausstattung mit materieller Infrastruktur sowie die wirtschaftsgeographische Lage und Siedlungsdichte gut bis sehr gut, jedoch ist hier die sektorale Struktur mit ihrer ausgeprägten Monofunktionalität im sekundären Bereich die weitaus wichtigere Determinante der Regionalentwicklung. In den östlichen Regionen Polens sind wirtschaftsgeographische Lage, Bevölkerungsdichte, Infrastrukturausstattung und Sektorstruktur als negativ zu bewerten.

Auch die Wanderungen der Faktoren Arbeit und Kapital begünstigten in der Transformationsphase die nationalen Zentren des Landes. Die Land-StadtWanderung trocknete das ohnehin schwache Bevölkerungspotential der ländlichen östlichen Regionen weiter aus. Dabei ist allerdings hervorzuheben, daß das beschränkte Wohnungsangebot in den Agglomerationen die Immigration in die Zentren gebremst hat. Ebenso wie der Faktor Arbeit, so ist während der 90er Jahre auch das Kapital in die Zentren gewandert. Ein sekundäres Zielgebiet stellten die westlichen Regionen des Landes dar. Hier ist die Nähe zu Deutschland als wichtiger Faktor hervorzuheben.

Was die Tauschkosten betrifft, läßt für gegenseitige Wirtschaftshilfe sich folgender Entwicklungsverlauf erkennen. Mit dem Zusammenbruch des Rates für gegenseitige Wirtschaftshilfe, der faktisch den polnischen Handel mit dem Rest der Welt unterbunden hat, ergab sich ein erster Schub durch die Integration in die Weltwirtschaft und insbesondere in den Handel mit der EU. Da zunächst die Güter der Transformationsländer auf dem Weltmarkt nicht konkurrenzfähig waren und eine hohe Nachfrage nach westlichen Gütern bestand, kam es zum Zusammen- 
bruch der Produktion. Mit dem Aufbau einer funktionierenden institutionellen Infrastruktur sanken nicht nur die Tauschkosten für den Handel, wie z. B. hohe Unsicherheit, Währungsrisiken usw., sondern auch für die Produktion. Da jedoch die Tauschkosten auch durch die Verkehrsanbindung und Kommunikationsmöglichkeiten bestimmt werden, entwickelten sich Agglomerationen und diejenigen Regionen, die an den Hauptverkehrsachsen zu dem größten Handelspartner (EU) liegen, am besten. Mit dem anvisierten Beitritt zur EU in der ersten Hälfte der nächsten Dekade werden sich diese Effekte weiter verstärken. Nivellierend könnte dann der Effekt auf der Basis der Williamson-Hypothese wirken, wenn im Zuge eines rasch ansteigenden BIP Mittel für die Erschließung der peripheren Räume in den Transformations-Ländern gesteigert sowie die Regionalpolitik intensiviert werden. Denn die Regionalpolitik spielt in der Anfangsphase der Transformation praktisch keine Rolle.

\section{Regionalpolitik}

Im Hinblick auf die Regionalpolitik während der Phase der Transformation sind grundsätzlich zwei Ebenen von Bedeutung: Inwieweit besteht eine nationale Regionalpolitik und inwieweit sind regionale gebietskörperschaftliche Ebenen in der Lage, eine eigenständige Regionalpolitik durchzuführen? Eine dritte Ebene bildet sich erst in der jüngsten Zeit heraus: die supranationale, d. h. die EU-Ebene.

\subsection{Nationale Regionalpolitik}

Die staatliche polnische Regionalpolitik der Transformationsphase von 1990 bis 1995 ist gekennzeichnet durch die Abwesenheit einer programmatischen und vorausschauenden Grundlage für eine langfristige regionale Entwicklungspolitik, eine unklare Kompetenzverteilung zwischen den zentralen Regierungsstellen und die Diskrepanz zwischen Zielen und institutionellen und finanziellen Ressour- cen. Inzwischen wurden jedoch erste Aktivitäten zum Aufbau einer nationalen Regionalpolitik eingeleitet. In Polen wurde dazu eine Task Force for Regional Development eingerichtet, die im Jahr 1996 Grundsätze einer staatlichen Regionalpolitik formuliert hat: Demnach soll die zukünftige polnische Regionalpolitik darauf ausgerichtet sein, eine hohes nationales Wirtschaftswachstum zu unterstützen, die Wettbewerbsfähigkeit polnischer Waren und Dienstleistungen im europäischen und globalen Maßstab zu erhöhen und Polen als Investitionsstandort für heimisches und ausländisches Kapital attraktiver zu machen. ${ }^{20}$ Ergänzt werden soll diese auf Effizienz und die nationalen Wachstumszentren zielende, zukünftige Regionalpolitik durch die Förderung der derzeit noch unterentwickelten Regionen, damit auch dort langfristig eine positive Entwicklung sichergestellt werden kann. Im Rahmen der zukünftigen regionalpolitischen Maßnahmen ist ein Instrumentenmix vorgesehen, der gängige, auch in westlichen Staaten angewandte Strategien einschließt. ${ }^{21}$

Bei der Aufstellung und Implementation der vorgeschlagenen Regionalpolitik in Polen werden verschiedene Prinzipien zugrunde gelegt: Subsidiarität zur Reduktion der nationalen Interventionen auf das notwendige Maß, soziale Partnerschaft zur Sicherstellung eines breiten Konsens über geplante Maßnahmen und ihre Realisierung, intergrierter Ansatz zur Realisierung von Synergieeffekten im ökonomischen, sozialen und ökologischen Bereich, Mittelkonzentration und Programmierung für eine langfristige Planungsgrundlage. ${ }^{22}$

Obgleich bereits 1996 neben den aufgezeigten Zielen nationaler Regionalpolitik auch deren institutionelle Ausgestaltung skizziert worden ist, bestehen bis heute Umsetzungsschwierigkeiten in diesem Bereich. Konkret zeigt sich, daß auf der nationalen Ebene nach wie vor keine Institution existiert, die alleine für regionalpolitische Belange verantwortlich ist. Das zentrale Planungsamt (CUP) und das Regierungszentrum für Strategische Studien
$(\mathrm{RCSS})^{23}$ bereiten zwar regional orientierte Politiken vor, sind aber im weiteren Prozeß nicht mit der Implementation der Strategien und Maßnahmen befaßt, da ihnen jegliche administrative Kompetenzen fehlen. ${ }^{24} \mathrm{Wie}$ instabil die regionalpolitische Situation auf der zentralen Ebene in Polen noch ist, zeigt das Beispiel des Anfang 1997 geschaffenen Regionalentwicklungskomitees, dem zwar weitreichende regionalpolitische Kompetenzen auf der nationalen Ebene zugewiesen wurden, dessen unzureichende institutionelle und finanzielle Ausstattung allerdings nur ein Jahr später wieder zu seiner Abschaffung führte. ${ }^{25}$

Derzeit wird über die Einrichtung eines Komitees für regionale und nachhaltige Entwicklung diskutiert, das die zentrale regionalpolitische Rolle auf der nationalen Ebene übernehmen soll. Der Zeitpunkt der Einrichtung steht allerdings noch nicht fest. Angesichts der Erfahrungen mit dem Regionalentwicklungskomitee des Ministerrates scheint der Erfolg eines weiteren, ähnlichen Komitees entscheidend davon abzuhängen, mit welchen Kompetenzen und Mitteln es ausgestattet sein wird. Des weiteren existiert ein Gesetzentwurf zur Regionalpolitik, der bei Fachleuten jedoch umstritten ist. ${ }^{26}$

Das polnische Beispiel deutet auf die beiden wesentlichen Tendenzen in bezug auf eine nationale Regionalpolitik in den MOEL in der Transformationsphase hin: Einerseits befindet sich die nationale Politikebene noch in der Phase der Umstrukturierung und der Neuverteilung von Kompetenzen. Andererseits werden die im Rahmen der Transformation zu bewältigenden Aufgabenstellungen als nationale Aufgaben gesehen, die zunächst kaum Raum für regionale Strategien lassen. Insofern sind in den meisten Ländern keine oder nur sehr schwache regionalpolitische Orientierungen zu erkennen.

\subsection{Regionalpolitik auf der regionalen Ebene}

Ein zweiter Ausgangspunkt für eine Regionalpolitik in den MOEL könnte 
auf der regionalen Ebene gesehen werden. Dies bedarf allerdings eigenständig verfaßter regionaler Gebietskörperschaften in diesen Ländern. Im Hinblick auf die institutionelle Struktur auf regionaler Ebene sind im Rahmen des Transformationsprozesses jedoch zwei gegenläufige Tendenzen beobachtbar. Nach der Öffnung wurden die Kompetenzen der lokalen Gebietskörperschaften in vielerlei Hinsicht aufgewertet, was im Einklang mit den Vorstellungen einer Demokratisierung steht. Hingegen kam es, bezogen auf regionale Verwaltungsebenen, zu einer Abwertung. ${ }^{28}$ Diese Entwicklung muß im Lichte der historischen Entwicklung und des Zweiten Weltkriegs gesehen werden. In einigen Transformationsländern leben ethnische Minoritäten mit unterschiedlichem geschichtlichen und kulturellen Hintergrund. Das sozialistische Wirtschaftssystem benötigte regionale Planungsregionen, wobei jedoch bei der Abgrenzung die kulturellen Unterschiede nicht berücksichtigt wurden. Nach der Öffnung der Systeme verloren diese Planungsregionen ihre Legitimation. Da nun Befürchtungen bestehen, daß eine an den historischen Gegebenheiten orientierte Abgrenzung von Regionen zusätzliche politische Spannungen erzeugen könnte, ist es sehr schwer, einen politischen Konsens über neue Regionsabgrenzungen $\mathrm{zu}$ erreichen..$^{29}$ In diesem Sinne muß in der Regel bei dem Regionsbegriff von statistischen Regionen ausgegangen werden. Insofern kann davon ausgegangen werden, daß in den MOEL von der regionalen Ebene mit der Ausnahme Polens bisher kaum Impulse für die Regionalentwicklung ausgegangen sind. Auf unterster Ebene erreichen offensichtlich auch die Gemeinden kaum Größenordnungen, die verwaltungstechnisch in der Lage sind, regionalpolitisch aktiv zu werden. Auf der Kreisbzw. Distriktebene dominiert zudem der Status einer staatlichen Verwaltungseinheit ohne eigene Entscheidungskompetenz, was die Abhängig keit von nationalen Initiativen verschärft.

Dies wird ebenfalls durch die im Rahmen der Agenda 2000 der Europäi- schen Kommission evaluierte nationale Regionalpolitik in den Bewerberländern bestätigt. Es zeigen sich kaum Ansatzpunkte, die sich für eine EURegionalpolitik nach dem bestehenden Muster - partnerschaftliche Institutionen, regionale Entwicklungskonzepte, Kofinanzierung - eignen. Diesen Erfordernissen kommt die Regionalpolitik in Polen, Slowenien und Ungarn noch am nächsten. ${ }^{30}$

Polen ist das erste Land unter den MOEL, das seinen territorialen Verwaltungsaufbau auf regionaler Ebene entscheidend reformiert hat (Abb. 4). Am 27. Juli 1998 unterzeichnete der polnische Präsident das Gesetz über die dreistufige territoriale Gliederung des Staates, das am 1. Januar 1999 in Kraft trat. Hiernach setzt sich das polnische Territorium aus neu abgegrenzten regionale (16) Wojewodschaften und der Kreis-Ebene (373 Powiats) zusammen. Die unterste Stufe, die lokale Ebene mit 2489 Gminas, wurde be-

\section{Abbildung 4}

Die polnischen Regionen nach dem 1. Januar 1999

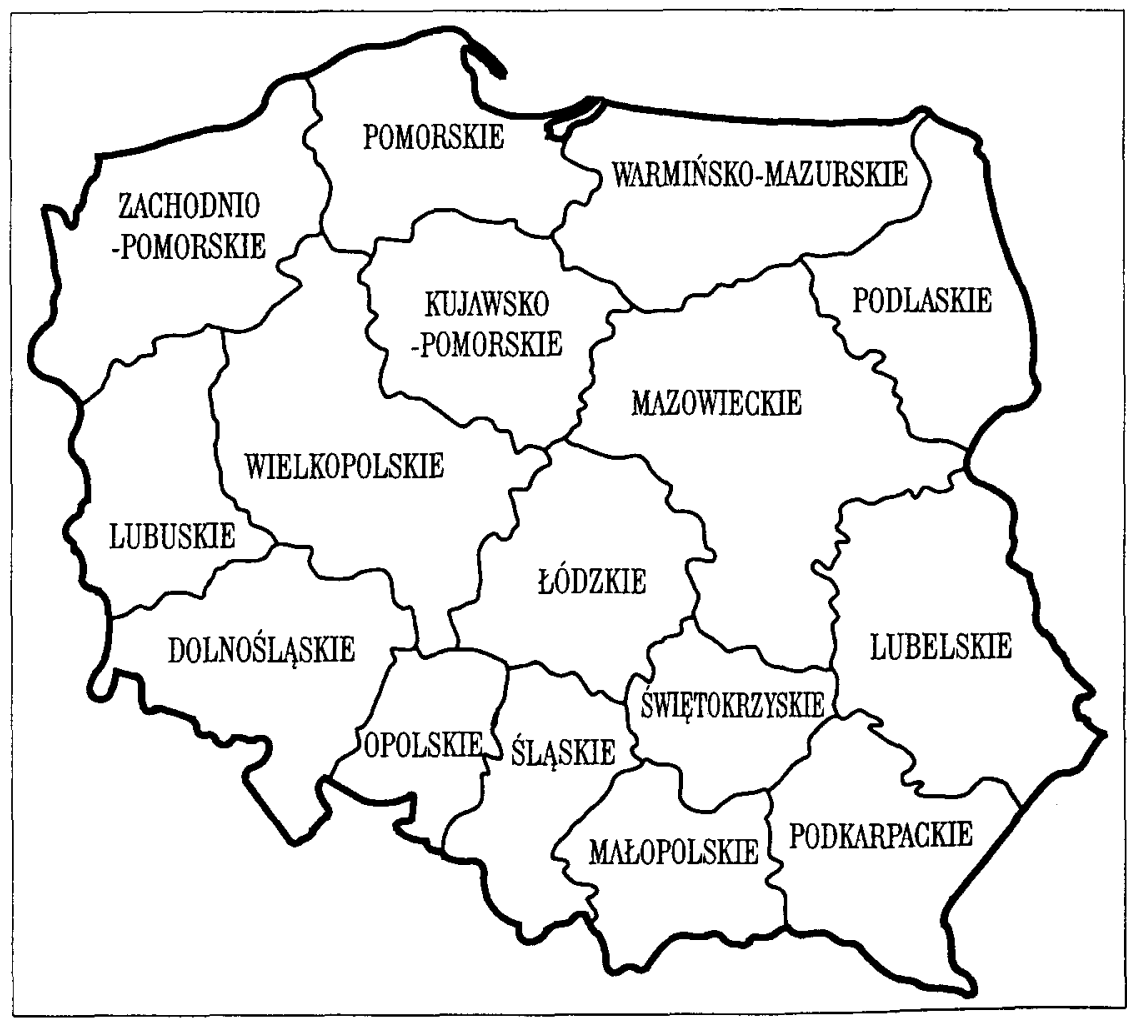

Quelle: Gorzelak 1998, S. 23 reits 1990 eingefuihrt und gilt als der erste Schritt der Territorialreform. ${ }^{31}$

Dem direkt gewählten Parlament der Wojewodschaft steht ein Marschall (Marsalek) vor. Im Unterschied zur passiven und zentral bestimmten Rolle der alten Wojewodschaften sollen die neu geschaffenen regionalen Einheiten eigene Entwicklungsstrategien aufstellen und diese dann auch hauptsächlich selbst finanzieren. Der institutionelle Mechanismus mit einem Vertreter der Zentralregierung in der Wojewodschaft soll garantieren, daß die regionalen Strategien im Einklang mit den staatlichen Richtlinien stehen (Tab. 4). ${ }^{37}$

Die Periode von 1999 bis 2001 soll der Bewertung und evtl. der Anpassung der neuen Territorialgliederung dienen. Hier soll sich aber an der Ebene der Wojewodschaften nichts ändern. Möglicherweise werden vereinzelt die Grenzen der Kreise (Powiat) den praktischen Anforderungen angepaßt. ${ }^{33}$ 
Tabelle 4

Charakteristika der neuen polnischen Regionen

\begin{tabular}{|ll|}
\hline Größe & - Durchschnittliche Fläche: $196000 \mathrm{~km}^{2}$ \\
& - Durchschnittliche Einwohnerzahl: 2,4 Mio. \\
\hline Hauptaufgaben & - Wirtschaftliche Entwicklung: Verbesserung der regionalen Wettbewerbs- \\
& und Innovationsfähigkeit, auch in internationaler Perspektive \\
& - Höherrangige öffentliche Dienstleistungen (z. B. höhere Bildung) \\
& und natürlichen Potentials, d. h. auch Landschaftsplanung und -nutzung \\
\hline Einnahmen & - Im wesentlichen durch regionale Zuschlagssätze auf Einkommens- bzw. \\
& Körperschaftssteuer \\
& - Ärmere Wojewodschaften sind förderungswürdig im Rahmen der \\
& Ausgleichssubvention \\
\hline Institutionelle & Sonstige Einnahmen, z. B. zweckgebundene zentrale Mittel \\
Struktur & Regionales Parlament (Sejmik) als Entscheidungsorgan bestimmt \\
die Wojewodschafts-Regierung mit deren repräsentativem Oberhaupt \\
(Marsalek)
\end{tabular}

Quelle: eigene Erstellung nach Chancellery of the Prime Minister of the Republic of Poland (1998), S. $23 \mathrm{ff}$.

Die wesentlichen Einnahmequellen der regionale Ebene in Polen sollen zukünftig Anteile an der Einkommenund Körperschaftssteuer sein. Diese Anteile berechnen sich anhand regionaler Zuschlagssätze. Wojewodschaften, die zu geringe eigene Einnahmen haben, sollen im Rahmen der Ausgleichssubventionen finanziell unterstützt werden (Tab. 4).

\subsection{Zunehmende Europäisierung der Regionalpolitik}

Im Zuge der Beitrittsbemühungen der MOEL in die EU wurden schon kurz nach dem Zusammenbruch der sozialistischen Systeme finanzielle Hilfsprogramme insbesondere im Rahmen des Phare-Programms und der Assoziierungsabkommen verabschiedet. 1997 wurde zudem von der Europäischen Kommission mit der Agenda 2000 ein Programm verabschiedet, das erste Schritte für ein Verfahren zum Beitritt und weitere finanzielle Hilfen im Rahmen einer Heranführungsstrategie definiert. Allerdings sehen sowohl das Phare-Programm als auch die Mittel aus der Heranführungsstrategie nur geringe Mittel für regionalspezifische Maßnahmen vor. ${ }^{34}$ Auch nach einem möglichen Beitritt, legt man die Ent- wicklung in den südeuropäischen Kohäsionsländern zugrunde, ist nicht zu erwarten, daß der regionale Faktor eine übergeordnete Rolle spielt. Dort flieBen zwar nicht pro Kopf, aber pro Region die größten Summen in die entwickelten Gebiete. Da kaum zu erwarten ist, daß bei der Osterweiterung das gegenwärtige Transferniveau aufrechterhalten werden kann, wird sich diese Tendenz verstärken.

Dennoch versucht die EU, die nationalen Regionalpolitiken der beitrittswilligen MOEL schrittweise an die EU-typischen strukturpolitischen Verfahrensweisen anzupassen. In Polen zählen hierzu beispielsweise Programme der grenzüberschreitenden Zusammenarbeit (polnisch-deutsche Euregios) sowie die von der Polnischen Regionalentwicklungsagentur (PARR) durchgeführten STRUDERProgramme. Die 1993 als Stiftung des Staatsschatzes gegründete PARR soll regionale Entwicklungsmaßnahmen vorbereiten, implementieren und beobachten. Hierzu fördert sie die Informationsdiffusion, Beratungsdienstleistungen, Trainingsprogramme, die Einrichtung von Finanzierungsinstitutionen und stellt finanzielle Mittel für die regionale Entwicklung zur Verfügung. Darüber hinaus kooperiert sie mit staatlichen Institutionen und
Nichtregierungsorganisationen, die sich mit Fragen der Regionalentwicklung und struktureller Arbeitslosigkeit befassen. Die PARR ist verantwortlich für die Implementierung von hauptsächlich Phare-Programmen in 17 Wojewodschaften und ist hinsichtlich der Programmdurchführung der Ansprechpartner der EU in Polen. Die Resultate und Erfahrungen sollen der polnischen Regierung helfen, umfassendere regionalpolitische Programme zu entwickeln und zu implementieren. Die regionalen Problemlagen reichen von schwach besiedelten und agrarisch dominierten Regionen (Olsztyn, Suwalki) bis hin zu altindustrialisierten Gebieten (Katowice, Lódz, Walbrzych) und Regionen, die durch eine duale Beschäftigungsstruktur (worker-farmers in Rzeszów) geprägt sind. Gemeinsames Merkmal aller Regionen ist die hohe offene oder verdeckte strukturelle Arbeitslosigkeit.

Im Hinblick auf das strukturpolitische Prinzip der Partnerschaft hat sich gezeigt, daß regionale Unterschiede im Qualifikationsniveau existieren. Das Qualifikationsniveau bezieht sich dabei auf ein grundsätzlich besseres Bildungsniveau, mehr Erfahrung im Umgang mit regionalpolitischen Maßnahmen und generell intensivere Beziehungen zu den Institutionen der EU. Bei den höher entwickelten Regionen bestehen zudem institutionelle Vorteile gegenüber den peripheren Regionen. ${ }^{35}$

\section{Schlußfolgerungen - Zukunftsperspektiven}

Die Niveauunterschiede im Hinblick auf die wirtschaftliche Leistungsfähigkeit zwischen den MOEL und allen Mitgliedstaaten der EU sind groß. Auch bei relativ hohen Wachstumsraten in den MOEL wird eine Angleichung der Niveaus auch mittelfristig nicht zu erwarten sein. Die MOEL haben den Transformationsschock mit dem Rückgang des BIP weitgehend überwunden und befinden sich in der Aufstiegsphase der sog. J-Kurve (siehe 
Abb. 1). Allerdings sind die Wachstumsraten bisher in einigen Ländern nicht sehr stabil, und auch die Transformation der Institutionen zur Marktwirtschaft sind noch nicht abgeschlossen, was zur Verlängerung des Anpassungs- und Aufholungsprozesses führen wird.

$\mathrm{Zu}$ Beginn der Transformationsphase in den MOEL war die Raumstruktur im Vergleich zu Westeuropa relativ ausgeglichen. Im weiteren Verlauf führte die Transformation zu einer Vergrößerung der Disparitäten zwischen den Regionen der MOEL. Dennoch liegen derzeit die regionalen Disparitäten in den MOEL im Vergleich zu den EU-Staaten immer noch auf einem geringeren Niveau. Auch zukünftig ist mit einer Zunahme der regionalen Disparitäten zu rechnen, da v. a. die Agglomerationen am günstigsten mit Potentialfaktoren ausgestattet sind, ihre Tauschkostenniveaus in $\mathrm{Re}$ lation zum Zentrum der EU am raschesten sinken, Hauptanziehungspunkte der Faktorwanderungen sind und darüber hinaus keine auf regionalen Ausgleich abzielende Regionalpolitik existiert.

Im Zuge einer Erweiterung der EU werden einzig die Hauptstadtregionen der MOEL ohne Schwierigkeiten im Binnenmarkt bestehen können, da die Agglomerationen über eine relativ gute Ausgangsbasis, was den Wohlstand und die Entwicklungsbedingungen betrifft, verfügen. Hier vollzieht sich die Transformation wesentlich schneller. Zudem zeichnet sich eine Arbeitsteilung zwischen den Metropolen der MOEL ab: Warszawa als Tor zum Osten auf der Achse BerlinMinsk-Moskau, Budapest als Finanzmetropole der MOEL, Prag als kulturelles Zentrum Mittel- und Osteuropas, Bratislava als das räumlich am nächsten zu einer westlichen Großstadt (Wien) gelegene Zentrum Mittel- und Osteuropas.

Als sekundäre Wachstumszentren entwickeln sich diejenigen Regionen an den wichtigsten Verkehrsachsen und Entwicklungskorridoren aufgrund des leichteren Zugangs $\mathrm{zu}$ ausländischem
Kapital. Hierzu zählen i. d. R. auch die westlich gelegenen Regionen der MOEL mit ihrer größeren Nähe zu westlichen Märkten.

Die Entwicklung der Gruppe der ärmeren und peripheren Regionen in den MOEL wird sich zukünftig aufspalten, so daß ein Zentrum der Peripherie und eine periphere Peripherie zu erwarten ist. Erste Beispiele hierfür sind schon erkennbar (Polen). Die erfolgreicheren Regionen dieser Gruppe können ihre Potentiale (z. B. landwirtschaftlich oder touristisch) zunehmend ausschöpfen. Ein wichtiger Faktor hierbei ist die Lage an den transeuropäischen Netzen (Helsinki-Korridore).

In den MOEL sind die Bedingungen für eine Regionalpolitik auf nationaler und auf regionaler Ebene bisher ungünstig, so daß kaum Impulse für eine eigenständige Regionalpolitik aus diesen Ländern zu erwarten sind. In bezug auf institutionelle Reformen auf regionaler Ebene ist Polen am weitesten fortgeschritten. Inwieweit die von einzelnen MOEL formulierten regionalpolitischen Ziele und Strategien erfolgreich in Maßnahmen und Projekte überführt werden können, ist derzeit noch nicht abzusehen.

Parallel zur Neuorientierung der nationalen Regionalpolitiken findet eine Europäisierung dieser Politiken statt; durch das regionalpolitische Engagement der EU werden erste Erfahrungen im Umgang mit EU-typischen strukturpolitischen Verfahren auf nationaler und regionaler Ebene gemacht. Dieses Learning-by-doing scheint eine wesentliche Voraussetzung für die effiziente Verwendung nationaler und auch in Zukunft strukturpolitischer EU-Mittel in den MOEL nach ihrem Beitritt zu sein.

Aus theoretischen Überlegungen sowie empirischen Erfahrungen aus der Süderweiterung Mitte der 80er Jahre kann gefolgert werden, daß folgende Faktoren für eine erfolgreiche Integration der mittel- und osteuropäischen Staaten von besonderer Bedeutung sind: ${ }^{36}$ Stabile und solide makroökonomische Rahmenbedingungen, wie z. B. geringe Inflation und geringes Staatsdefizit, um die Unsicherheit für den Aufbau von Unternehmen und beim Handel zu verringern, sowie der Ausbau der Infrastruktur in allen Bereichen und insbesondere des Verkehrs und der Telekommunikation, um den Standort nicht nur als Absatz-, sondern auch als Produktionsstandort attraktiv zu machen. Eine der wirtschaftlichen Kohäsion verpflichtete Förderpolitik der EU muß sich deshalb zunächst auf eine Heranführung der Beitrittsländer aus den MOEL an das EU-Niveau konzentrieren, wobei regionale Gesichtspunkte auf nationaler Ebene in der ersten Phase eine untergeordnete Rolle spielen werden. Insofern können die Entwicklungsaussichten der schwachen Regionen in den MOEL auch auf mittlere Sicht nicht positiv bewertet werden, wohingegen die starken Regionen den Anschluß an des EUNiveau schaffen werden.

\section{Anmerkungen}

(1)

European Commission (Ed.): Agenda 2000, Vol. I and Vol. II - Communication: The effects on the Union's policy of Enlargement to the Applicant Countries of Central and Eastern Europe (Impact Study). - Brussels 1997, S. 80

\section{(2)}

European Commission (Ed.): First Report on Economic and Social Cohesion - Brussels 1996, S. 18. Bei den Kohäsionsländern konnte eine Abnahme der Disparitäten im Vergleich zum EU-Durchschnitt seit 1983 festgestellt werden, wobei sich hier für die einzelnen Länder sehr unterschiedliche Werte ergeben haben: Aufgrund unterschiedlicher nationaler Wachstumsraten holte Irland z. B. bis 1995 um 29, Portugal um 13, Spanien um 7 und Griechenland um 2 Prozentpunkte auf (1996, S. 18). Dies zeigt, daß im Rahmen der europäischen Integration ein AufholprozeB innerhalb eines absehbaren Zeitraums möglich, nicht aber zwangsläufig gegeben ist.

(3)

European Bank for Reconstruction and Development (EBRD) (Ed.): Transition Report. London 1996, S. 12

(4)

Balcerowicz, L.: Prescriptions for Poland. Shock Therapy Played its Part, but Sustained Growth Requires Deeper Measures. In: Central and Eastern European Review, Warsaw (1998), July/August, S. 8 
(5)

Die Bewertungsskala der European Bank for Reconstruction and Development (EBRD) reicht von 1 bis 4+, wobei eine Bewertung von $4+$ entwickelten Volkswirtschaften entspricht.

(6)

Hallet, M.: National and Regional Development in Central and Eastern Europe: Implications for the Structural Assistance. - Brussels 1997. In: European Commission, Directorate-General for Economic and Financial Affairs (Ed.): Economic Papers, No, 120, S. 13. Aufgrund statistischer Probleme können diese Daten allenfalls dazu verwendet werden, Tendenzen aufzuzreigen. Sie berücksichtigen zudem keine regionale Preisdifferenzen.

(7)

European Commission (Ed.): First Report ..., a. a. O. [siehe Anm. (2)], S. 24

(8)

European Policy Research Centre (EPRC); Rheinisch-Westfälisches Institut für Wirtschaftsforschung (RWI) (Ed.): The Impact on Cohesion of EU Enlargement - Draft Final Report to the European Commission (DG XVI). Strathclyde, Essen 1996. Grundlage der Berechnungen sind die am Durchschnitt des jeweiligen Landes normierten Werte.

(9)

Das Verhältnis von der reichsten zur ärmsten Region (Wojewodschaft) betrug 1986 2,9:1 und ist bis 1992 auf 2,5:1 gesunken.

(10)

Gorzelak, G.: Regional and Local Potential for Transformation in Poland. - Warsaw 1998. $=$ Regional and Local Studies, 14, S. 65 f.

\section{(11)}

Unter der relativen Wachstumsrate ist die $\mathrm{Zu}$ oder Abnahme des regionale BIP/EW in Relation zum jeweiligen nationalen Durchschnitt $(=100)$, d. h. für die Perioden von 1986 bis 1992 und von 1992 bis 1995 , zu verstehen.

(12)

Biehl, D.; Ungar, P.: Disparitäten, regionale. In: Akademie für Raumforschung und Landesplanung (ARL) (Hrsg.): Handwörterbuch der Raumordnung. - Hannover 1995, S. 185-189

(13)

Biehl, D.: Infrastruktur als Bestimmungsfaktor regionaler Entwicklungspotentiale in der Europäischen Union. In: Karl, H.; Henrichsmeyer, W. (Hrsg.): Regionalentwicklung im Prozeß der Europäischen Integration. - Bonn 1995. = Bonner Schriften zur Integration Europas, Bd. 4, S. $59 \mathrm{ff}$.

\section{(14)}

Eigene Übersetzung von Williamson, J. G.: Regional Inequality and the Process of National Development: A Description of the Patterns. In: Economic Development and Cultural Change, Washington, 13. Jg. (1965), S. 9

(15)

Vgl. ebenda, S. $5 \mathrm{ff}$.
(16)

Krugman, P. R.; Venables, A. J.: Integration and the Competitiveness of Peripheral Industry. In: Bliss, C.; Braga de Macedo, J. (Ed.): Unity and Diversity in the European Economy: The Community's Southern Frontier. - Cambridge 1990; Krugman, P.: Geography and Trade. Leuven 1991

(17)

Ausführlich Eser, T. W.; Hallet, M.: Der mögliche Beitrag der EG-Regionalpolitik bei einer Ost-Erweiterung: Hilfe oder Hindernis? In: Osteuropa - Wirtschaft, 38. Jg. (1993), S. $197 \mathrm{ff}$ und Hallet, M.: Wirkungen wirtschaftlicher Integration auf periphere Regionen. - Trier 1997 $=$ Trierer Schriften zur Wirtschaftstheorie und Wirtschaftspolitik

(18)

Hallet, M.: Wirkungen wirtschaftlicher Integration auf periphere Regionen, a. a. O. [siehe Anm. (17)], S. 11

(19)

Abraham, T.: Regionalentwicklung in Mittelund Osteuropa im Spannungsfeld von Transformation und Integration - dargestellt am Beispiel Polens. - Trier 1998. = Diplomarbeit an der Universität Trier im Fachbereich Geographie/ Geowissenschaften

\section{(20)}

Task Force for Regional Development in Poland (Ed.): Outline of a Regional Development Strategy for Poland (Final Report). - Warsaw 1996, S. 7 ff. Hauptaufgabe der im Jahre 1995 gemeinsam von der polnischen Regierung und der EU eingerichteten Task Force war es, eine regionale Entwicklungsstrategie aus der nationalen Perspektive zu entwerfen.

(21)

Vgl. ebenda, S. $7 \mathrm{ff}$. Es werden die Bereiche Humankapital, Innovation, endogenes Potential, Technologie, Infrastruktur und Produktives Umfeld betont.

Vgl. ebenda, S. 8

(23)

Das am 31. November 1996 eingerichtete Regierungszentrum für Strategische Studien ersetzte praktisch das Zentrale Planungsamt (CUP).

\section{(24)}

Gorzelak, G.: Regional and Local Potential..., a. a. O. [siehe Anm. (10)], S. 164 f

\section{(25)}

Task Force for Structural Policy in Poland (Ed.): Structural Policy in Poland from the Perspective of Integration with the European Union (Final Report). - Warsaw 1998. = Regional and Local Studies, 14, S. 165

\section{(26)}

Abraham, T.: Regionalentwicklung in Mittelund Osteuropa ..., a. a. O. [siehe Anm. (19)], S. 89
(27)

Eser, T.W.: Effects of an Enlargement of the EU Towards the East on the Regions of the Accession Countries. - Birmingham 1998. = Discussion Papers in German Studies, No. IGS 98/7, S. 17

\section{(28)}

Hallet, M.: National and Regional Development ..., a. a. O. [siehe Anm. (6)], S. $3 \mathrm{f}$.

(29)

Vgl. ebenda

(30)

European Commission (Ed.): Agenda 2000 Commission Opinion on the Application for Membership of the European Union (of the ten Central and Eastern European Countries). Brussels 1997

(31)

Chancellery of the Prime Minister of the Republic of Poland (Ed.): Effectiveness, Openess, Subsidiarity. A New State for New Challenges. - Warsaw 1998, S. 5 ff.

\section{(32)}

Vgl. ebenda, S. 23 ff. Der Finanzierungsmechanismus liegt bislang nur als Gesetzentwurf vor.

(33)

Vgl. Abraham, T.: Regionalentwicklung in Mittel- und Osteuropa ..., a. a. O. [siehe Anm. (19)], S. 91

\section{(34)}

Eser, T. W.: Effects of an Enlargement of the EU . . . a. a. O. [siehe Anm. (27)], S. 46

(35)

Vgl. Abraham, T.: Regionalentwicklung in Mittel- und Osteuropa . . , a. a. O. [siehe Anm. (19)], S. 75

(36)

Hallet, M.: Wirkungen wirtschaftlicher Integration ..., a. a. O. [siehe Anm. (17)]; Larre, B.; Torres, R.: Is Convergence a Spontaneous Process? The Experience of Spain, Portugal and Greece. - Paris 1991. In: OECD Economic Studies, Bd. 16

Dipl.-Geogr. Thomas Abraham PROGNOS GmbH

Unter Sachsenhausen 37

50667 Köln

E-Mail: thomas.abraham@prognos.com.de

Dr. Thiemo W. Eser

TAURUS Institut an der Universität Trier Universitätsring 15 54286 Trier

E-Mail: eser@uni-trier.de 